\title{
Estimating the Domain of Attraction based on the Invariance Principle
}

\author{
Dongkun Han, Ahmed El-Guindy, and Matthias Althoff
}

\begin{abstract}
Estimating the domain of attraction (DA) of an equilibrium point is a long-standing yet still challenging issue in nonlinear system analysis. The method using the sublevel set of Lyapunov functions is proven to be efficient, but sometimes conservative compared to the estimate via invariant sets. This paper studies the estimation problem of the DA for autonomous polynomial system by using the invariance principle. The main idea is to estimate the DA via sublevel sets of a positive polynomial, which characterizes the boundary of invariant sets. This new type of invariant sets admits the condition that the derivative of Lyapunov functions is non-positive, which generalizes the sublevel set method via Lyapunov functions. An iterative algorithm is then proposed for enlarging the estimate of the DA. Finally, the effectiveness of the proposed method is illustrated by numerical examples.
\end{abstract}

\section{INTRODUCTION}

For a given locally asymptotically stable equilibrium point, the domain of attraction (DA) is a set of initial states for which all trajectories starting from this region converge to the equilibrium point. By estimating the DA, one can directly predict the stability and the safety margin of systems with respect to a given initial state. Since long time ago, estimating the DA has always been closely related to engineering implementations, e.g., transient stability analysis of power systems and chemical reaction prediction [1]. More recently, its implementation has expanded quickly into the areas of clinical immunotherapy, ecological economy and biological systems, just to name a few [2]. For more applications, please check the survey [1] and the book [3].

Amongst the pioneering explorations, Zubov proposed a necessary and sufficient condition for the existence of Lyapunov function with respect to the DA of an equilibrium point [4]. This condition enables us to obtain the exact DA by solving a partial differential equation, which is called Zubov equation (also see its extensions: Zubov-like equation or Zubov generalized equation [5]). Based on [4], a new concept of maximal Lyapunov functions was defined: It is characterized by a partial differential equation and can be approximated by rational polynomial functions [6]. These methods can provide the actual DA, whereas they have to calculate the solution of Zubov-like equations, which is generally not easy to obtain. In order to avoid solving the Zubov equation, some effective methods have been proposed. These methods can be roughly classified into two categories: Lyapunov methods and non-Lyapunov methods.

The authors are with the Institut für Informatik, Technische Universität München, Boltzmannstraße 3, 85748 Garching bei, München, Germany. E-mail: $\{$ hand, elguindy, althoff $\}$ @in.tum.de
Lyapunov methods exploit the sublevel set of Lyapunov functions for constructing an invariant and contractive set to inner-approximate the DA of the equilibrium point. This method has been widely studied in the last decade, thanks to the progress of real algebraic geometry [7] and positive polynomials [8], especially the sum-of-squares (SOS) technique [9]-[13]. Various effective approaches have been proposed by using different sublevel sets of distinct types of Lyapunov functions [14]-[22]. Quadratic Lyapunov functions approximate the DA efficiently, and conditions of Linear Matrix Inequalities (LMIs) are proposed by computing the Lyapunov matrix [20], [21]. In [18], a local triangulation strategy was proposed for two-dimensional systems via piecewise affine Lyapunov functions. In [17], polyhedral Lyapunov functions are constructed for quadratic systems. In [14], SOS programming conditions are proposed by using polynomial Lyapunov functions. Based on [14], pointwise maximum Lyapunov functions are formulated by searching feasible polynomial Lyapunov functions [15]. Also based on [14], the DA can be estimated by a union of sublevel sets by using a continuous family of polynomial Lyapunov functions [16]. A more recent work used rational polynomial Lyapunov functions and obtained less conservative results compared to polynomial ones [19].

Non-Lyapunov methods, by contrast, do not use the sublevel set of Lyapunov functions [23]-[26]. In [23], an outerapproximation of the DA is computed by a primal infinite dimensional linear programming (LP) problem, which can be further transformed into the dual problem of SOS programming by using the moment theory. Extended from [23], a convex approach has been proposed to compute the innerapproximation of the DA by alternatively over-approximating the complement of the DA [24]. Very recently, a new approach was proposed to estimate the DA of polynomial systems by using invariant sets in contrast to sublevel sets of Lyapunov functions [26]: An iterative algorithm was provided based on SOS programming. This method obtains less conservative results by using invariant sets, but it still demands a Lyapunov function to formulate the invariant set.

Inspired by the work in [26], and based on the work in [3], [27] in the category of Lyapunov method, this paper uses invariant principles to inner-approximate the DA without searching for a Lyapunov function. The main contributions of this paper are listed as follows:

- An approach is proposed for computing the largest estimate of the DA for nonlinear systems based on the Barbashin-Krasovskii-LaSalle principle. A new type of invariant set, as the sublevel set of a family of positive 
polynomials, is proposed to be the inner-approximation of the DA (Section III-A).

- Based on the real Positivstellensatz, the estimation problem of the DA can be reduced to a local SOS programming problem. By further exploiting the Square Matrix Representation (SMR) of local SOS, we can compute a lower bound of the largest estimate of the DA. This can be achieved by solving a generalized eigenvalue problem (Section III-B and Section III-C).

- Two strategies are proposed for computing the largest estimate of the DA with variable polynomials, which characterize the boundaries of invariant sets. Based on these strategies, an algorithm is given for enlarging the estimate of the DA via invariant sets (Section III-D).

\section{PRELIMINARIES}

Notations: $\mathbb{N}, \mathbb{R}$ : natural and real number sets; $\mathbb{R}_{+}$: positive real number set; $0_{n}$ : origin of $\mathbb{R}^{n} ; \mathbb{R}_{0}^{n}: \mathbb{R}^{n} \backslash\left\{0_{n}\right\} ; A^{T}$ : transpose of $A ; A>0(A \geq 0)$ : symmetric positive definite (semidefinite) matrix $A ; A \otimes B$ : Kronecker product of matrices $A$ and $B ; \operatorname{deg}(f)$ : degree of polynomial function $f ; \operatorname{trace}(A)$ : trace of matrix $A ; \operatorname{diag}(v)$ : a square diagonal matrix with the elements of vector $v$ on the main diagonal; $(*)^{T} A B$ in a form of SMR: $B^{T} A B$. Let $\mathcal{P}$ be the set of polynomials and $\mathcal{P}^{n \times m}$ the set of matrix polynomials with dimension $n \times m$. A polynomial $p(x) \in \mathcal{P}$ is nonnegative if $p(x) \geq 0$ for all $x \in \mathbb{R}^{n}$. A useful way of establishing $p(x) \geq 0$ consists of checking whether $p(x)$ can be described as a sum of squares of polynomials (SOS), i.e., $p(x)=$ $\sum_{i=1}^{k} p_{i}(x)^{2}$ for some $p_{1}, \ldots, p_{k} \in \mathcal{P}$. The set of SOS polynomials is denoted by $\mathcal{P}^{\mathrm{SOS}}$. If $p(x) \in \mathcal{P}^{\mathrm{SOS}}$ becomes 0 only for $x=0_{n}$, we call $p(x)$ local SOS denoted by $\mathcal{P}_{0}^{\text {SOS }}$.

\section{A. Model Formulation}

In this paper, we consider the following autonomous dynamical system:

$$
\dot{x}(t)=f(x(t)),
$$

where $x \in \mathbb{R}^{n}$ is the state vector, $x(0) \in \mathbb{R}^{n}$ is the initial state, $\chi(t ; x(0))$ denotes the solution of system (1), $f$ : $\mathbb{R}^{n} \rightarrow \mathbb{R}$ is a nonlinear function satisfying the local Lipschitz condition. In the sequel, we will omit the arguments $t$ and $x$ of functions whenever possible for the brevity of notations.

Remark 1: In this paper, we are interested in estimating the DA of an isolated stable equilibrium point. Without loss of generality, we place the equilibrium point at the origin.

Let us introduce the DA of the origin: It is the set of initial states for which the system asymptotically converges to the origin, i.e.,

$$
\mathcal{D}=\left\{x(0) \in \mathbb{R}^{n}: \lim _{t \rightarrow+\infty} \chi(t ; x(0))=0_{n}\right\},
$$

where $\chi$ is the solution of system (1). The set $\mathcal{I}$ is invariant if $x(0)=\chi(0 ; x(0)) \in \mathcal{I} \Rightarrow \chi(t ; x(0)) \in \mathcal{I}, \forall t \in \mathbb{R}$. If this condition only holds for $t \geq 0$, then $\mathcal{I}$ is positive invariant.

\section{B. Problem Formulation}

In this paper, we aim to use the invariant set to estimate the DA of the origin. Since the sublevel set method of Lyapunov functions is the dominating method in this area, we first introduce this method and then compare it with the method via invariant sets. Specifically, let $V(x)$ be a Lyapunov function of system (1) for the origin, which satisfies $\forall x \in$ $\mathbb{R}_{0}^{n}: V(x)>0, V\left(0_{n}\right)=0, \lim _{\|x\| \rightarrow \infty} V(x)=\infty$ and the time derivative of $V(x)$ along the trajectories of (1) is locally negative definite [28]. We introduce the sublevel set of $V(x)$ as

$$
\mathcal{V}(c)=\left\{x \in \mathbb{R}^{n}: V(x) \leq c\right\},
$$

where $c \in \mathbb{R}_{+}$. For system $(1), \mathcal{V}(c)$ is an estimate of $\mathcal{D}$ if there exists a positive scalar $c$ such that $\forall x \in \mathcal{V}(c) \backslash$ $\left\{0_{n}\right\}: \dot{V}(x)<0$ [28].

An important observation in [28] is that the sublevel set of a Lyapunov function $\mathcal{V}(c)$ for estimating the DA is simple but usually conservative compared to the method of invariant sets based on LaSalles's invariance theorem. This motivates us to find an invariant set which is more general and thus less conservative than the set $\mathcal{V}(c)$ to estimate the DA. See Fig. 1 for the relationship of different methods.

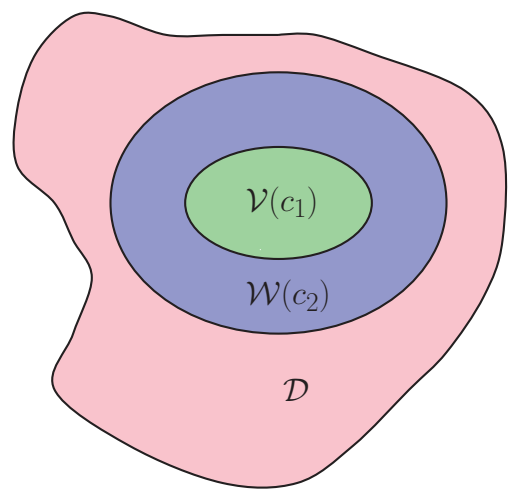

Fig. 1. An illustration for estimating the DA by using different methods The light pink area stands for the DA. The blue area indicates the innerestimate of the DA by using invariant sets. The green area indicates the inner-estimate of the DA by using the sublevel set of Lyapunov functions.

Let us now propose the main problem we are concerned with: Find a function $W(x)$ and a positive scalar $c$ such that

$$
\mathcal{W}(c)=\left\{x \in \mathbb{R}^{n}: W(x) \leq c\right\}
$$

is an invariant set, and the estimate of the DA is maximized under a certain selected criteria, i.e., by solving

$$
\begin{aligned}
& \mu=\sup _{c, W} \rho(\mathcal{W}(c)) \\
& \text { s.t. }\left\{\begin{array}{l}
\mathcal{W}(c) \subseteq \mathcal{D}, \\
\mathcal{W}(c) \text { is a positive invariant set, }
\end{array}\right.
\end{aligned}
$$

where $\rho$ is a measure of $\mathcal{W}(c)$ depicting the user-defined criteria. It is worth noting that problem (4) is an innerapproximation problem of the DA, which is non-convex due to the construction of invariant sets and the high nonlinearity of $\rho$. 


\section{MAIN RESUlts}

In this section, we first provide a method to estimate the DA via a new type of invariant set. Then, we execute our method by an iterative algorithm, in which each step will be introduced by each subsection.

\section{A. Estimate via the Invariant Principle}

First, the following invariant principle is introduced:

Lemma 1 (Barbashin's theorem [28]): Let $x=0_{n}$ be an equilibrium point for (1) and let $W(x)$ be a continuously differentiable positive definite function on a domain $\mathcal{A}$ containing the origin, such that $\dot{W}(x) \leq 0$. Let

$$
\mathcal{S}=\{x \in \mathcal{A} \mid \dot{W}(x)=0\}
$$

and suppose that no other solution can stay identically in $\mathcal{S}$, other than the trivial solution $x \equiv 0_{n}$. Then, the origin is locally asymptotically stable.

If the function $W$ in Lemma 1 is assumed to be a continuously differentiable, radially unbounded, and a positive definite function, the conclusion of Lemma 1 becomes: The origin is globally asymptotically stable. This turns out to be the Krasovskii's theorem [28]. Note that Barbashin's theorem and Krasovskii's theorem are specializations of LaSalle's theorem for the case of a single equilibrium point.

Remark 2 (Difference from Lyapunov stability theory):

The Barbashin-Krasovkii theorem requires that $\dot{W}(x) \leq 0$, while the Lyapunov function $V(x)$ has to satisfy the more strict condition that $\dot{V}(x)<0, \forall x \in \mathcal{A} /\left\{0_{n}\right\}$. In other words, the Lyapunov stability condition is a special case of the Barbashin-Krasovkii invariance theorem. However, one needs to establish that the maximum invariant set in $\mathcal{S}$ of (5) is the origin, which may not be simple in general.

In order to overcome this obstacle, a sufficient condition is provided by establishing the higher-order derivative of $W(x)$ and a new type of invariant set is developed for estimating the DA.

Theorem 1: Let $W(x): \mathbb{R}^{n} \rightarrow \mathbb{R}_{+}$be a positive definite analytic function on $\mathcal{W}(c)=\left\{x \in \mathbb{R}^{n} \mid W(x) \leq c\right\}$ and $W\left(0_{n}\right)=0$. If there exists a natural number $k$ such that

$$
\left\{\begin{array}{l}
W^{(2 k+1)}(x)<0, \forall x \in \mathcal{W}(c) /\left\{0_{n}\right\} \\
W^{(j)}(x)=0, j=0,1,2, \ldots, 2 k
\end{array}\right.
$$

where $W^{(0)} \equiv 0$ and $W^{(j)}$ is the $j$-th time derivative of $W$ with respect to $t^{1}$, then, $\mathcal{W}(c)$ is an estimate of the DA, i.e., $\mathcal{W}(c) \subseteq \mathcal{D}$.

Proof: We first prove that 1) $\mathcal{W}(c)$ is an invariant set: Then we show that 2) the origin is the only positive invariant set in $\{x \in \mathcal{W}(c) \mid \dot{W}=0\}$.

1) $\mathcal{W}(c)$ is an invariant set: Since $W(x)$ is positive definite on $\mathcal{W}(c) /\left\{0_{n}\right\}$, then $\mathcal{W}(c)$ is compact. According to [1, Theorem 3.1], and taking into account the assumption that $f$

\footnotetext{
${ }^{1}$ For example, $W^{(1)}(x)=\langle\nabla W, f(x)\rangle$ where $\langle\cdot, \cdot\rangle$ is the inner product of two vectors, $\nabla W$ is the gradient of $W$, i.e., $\nabla W=\left(\frac{\partial W}{\partial x_{1}}, \ldots, \frac{\partial W}{\partial x_{n}}\right)$.
}

satisfies the local Lipschitz condition, one can conclude that system (1) has a unique solution for all $x(0) \in \mathcal{W}(c)$. For the continuity of $\chi(t, x(0))$, it follows that

$$
\dot{W}(x(t)) \leq 0 \Rightarrow W(x(t)) \leq W(x(0)) \leq c, \forall t \geq 0 .
$$

Thus, all the solutions $\chi(t, x(0))$ remain in the set $\mathcal{W}(c)$.

2) The origin is the only positive invariant set in $\{x \in$ $\mathcal{W}(c) \mid \dot{W}=0\}$ : This condition holds if we can show that $0_{n}$ is locally asymptotically stable for all $x \in \mathcal{W}(c)$. Since $W(x)>0$ for all $\mathcal{W}(c) /\left\{0_{n}\right\}$, we only need to prove that under condition (6), the statement $\lim _{t \rightarrow \infty} W(x)=0$ holds, which directly yields $\lim _{t \rightarrow \infty} x=0$. This can be proven by using a contradiction. Let us assume that $\lim _{t \rightarrow \infty} W(x)=a$ and $a \neq 0$, and that there exist a time $T$ and a set $\mathcal{B}$ as a neighborhood of $0_{n}$ such that $\chi(t, x(0))$ is not in $\mathcal{B}$ for all $t \geq T$.

Define the sets $\mathcal{C}=\{x \in \mathcal{W}(c) \mid \dot{W}(x)=0\}$ and $\mathcal{E}=$ $\mathcal{C} / \mathcal{B}$. From condition (6), one could assume that there exists a $k$ such that

$$
\left\{\begin{array}{l}
\max _{x \in \mathcal{E}} W^{(2 k+1)}(x)=-\gamma, \forall x \in \mathcal{W}(c) /\left\{0_{n}\right\} \\
W^{(j)}(x)=0, j=0,1,2, \ldots, 2 k
\end{array}\right.
$$

where $\gamma$ is a positive scalar. By integrating $W^{(2 k+1)}$ with respect to the time interval $[t, T]$, one has

$$
\begin{aligned}
W(x(t))-W(x(T)) & =\underbrace{\int_{T}^{t} \ldots \int_{T}^{t}}_{2 k+1} W^{(2 k+1)} \mathrm{d} s \\
& \leq \underbrace{\int_{T}^{t} \cdots \int_{T}^{t}(-\gamma) \mathrm{d} s}_{2 k+1} \\
& =-\frac{\gamma(t-T)^{2 k+1}}{(2 k+1) !},
\end{aligned}
$$

which results in $W(x(t)) \leq W(x(T))-\frac{\gamma(t-T)^{2 k+1}}{(2 k+1) !}$. As $t$ goes to infinity, one has that $\lim _{t \rightarrow \infty} W(x)=-\infty$, which contradicts with the fact that $W(x)$ is positive definite on $\mathcal{W}(c) /\left\{0_{n}\right\}$. Thus, from Lemma $1,0_{n}$ is locally asymptotically stable for all $x \in \mathcal{W}(c)$, which ends this proof.

Remark 3: For Theorem 1, it is worth noting that

- The sublevel set method of Lyapunov functions can be deemed as a special case of the proposed method. By setting $k=0$ in (6), $W(x)$ turns out to be a Lyapunov function of (1) and $\mathcal{W}(c)$ is a sublevel set of a Lyapunov function, which is also a contractive and invariant set.

- Observe that Theorem 1 only provides a sufficient condition that enables the origin to be the maximum invariant set in $\mathcal{S}$ of (5). The conservatism originates from the fact that Theorem 1 holds only for $W(x)$ with the highest even derivative $W^{(j)}=0$. Another source of conservatism is that, as mentioned in [28], one could construct an invariant set $\mathcal{W}(c)$ while the function $W(x)$ is not necessarily positive definite. Methods for reducing the conservatisms will be discussed in Section V. 


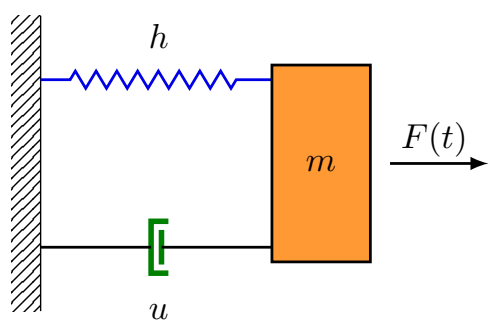

Fig. 2. The prototypical single degree of freedom system.

For the above result, a deliberately simple example is given for illustration:

Example 1 (An excitation-free vibratory system [29]): A spring-mass-damper model is considered in Fig. 2, where

$$
\begin{aligned}
x_{1}(t) & :=\text { displacement of the center of mass, } \\
x_{2}(t) & :=\dot{x}_{1}(t), \text { i.e., velocity of the object, } \\
F(t) & :=\text { external excitation force, } \\
m & :=\text { mass of the object, } \\
h & :=\text { elastic stiffness coefficient, } \\
u & :=\text { viscous damping coefficient. }
\end{aligned}
$$

For an excitation-free system, $F(t)=0$ and $m, h, u \in \mathbb{R}_{+}$. From Newton's second law of motion, one has

$$
\begin{aligned}
& \dot{x}_{1}(t)=x_{2}(t), \\
& \dot{x}_{2}(t)=-\frac{u}{m} x_{2}(t)-\frac{h}{m} x_{1}(t) .
\end{aligned}
$$

Let $x=\left(x_{1}, x_{2}\right)^{T}$, we first consider the Lyapunov function candidate $V_{1}(x)=0.5 h x_{1}^{2}+0.5 m x_{2}^{2}$. In order to check the stability of this system and compute the DA, the derivative of $V_{1}$ is: $\dot{V}_{1}(x)=-u x_{2}^{2}$. It yields that $\dot{V}_{1}=0$ for all the states on the $x_{2}$-axis. Based on Lyapunov stability theory, the origin is not guaranteed to be stable. Thus, no sublevel set of $V_{1}$ can be used as the estimate of the DA.

In contrast, we consider $W_{1}(x)=V_{1}(x)$ by using Theorem 1. Provided that $\dot{W}_{1}(x)=W_{1}^{(2)}(x)=0$, one has $x_{2}=0$ and $W_{1}^{(3)}=-\frac{u h^{2}}{m^{2}} x_{1}^{2}<0, \forall x \in\left\{x \mid x_{2}=0\right\} /\left\{0_{2}\right\}$. Therefore the global stability of the origin is ensured by using the proposed method. In addition, $W(x)$ satisfying condition (6) is usually not unique. One could also consider $W_{2}(x)=0.25 h x_{1}^{4}+0.25 m x_{2}^{4}$ with $\dot{W}_{2}(x)=W_{2}^{(2)}(x)=$ $W_{2}^{(3)}(x)=W_{2}^{(4)}(x)=0$. It yields that $x_{2}=0$ and $W_{2}^{(5)}=-\frac{24 u h^{4}}{m^{4}} x_{1}^{4}<0, \forall x \in\left\{x \mid x_{2}=0\right\} /\left\{0_{2}\right\}$. Thus, the estimates of the DA can be obtained via some level sets of $W_{1}(x)$ and $W_{2}(x)$, as shown in Fig. 3.

For the ease of understanding, we provide a flowchart of the iterative algorithm in Fig. 4, where Step 1 enlarges the estimate of the DA with fixed $W(x)$ by solving an SOS programming problem; Step 2 converts this problem to a quasi-convex optimization problem; Step 3 searches for the optimal $W(x)$ that provides the largest estimate of the DA with fixed auxiliary functions $r(x)$ and $s(x)$, which will be introduced in the next subsection.

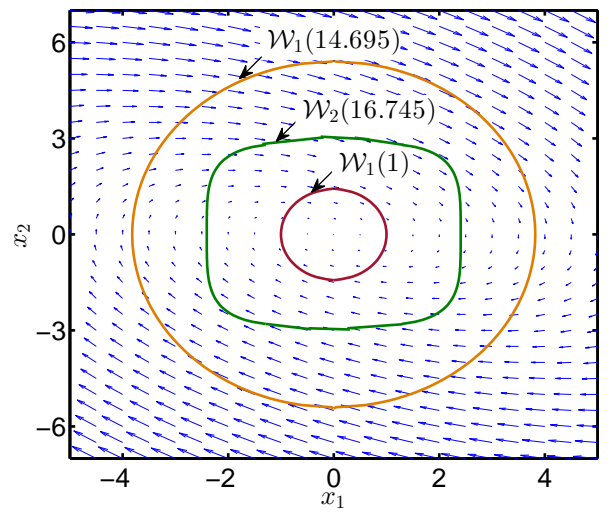

Fig. 3. The estimates of the DA by using $W_{1}(x)$ and $W_{2}(x)$.

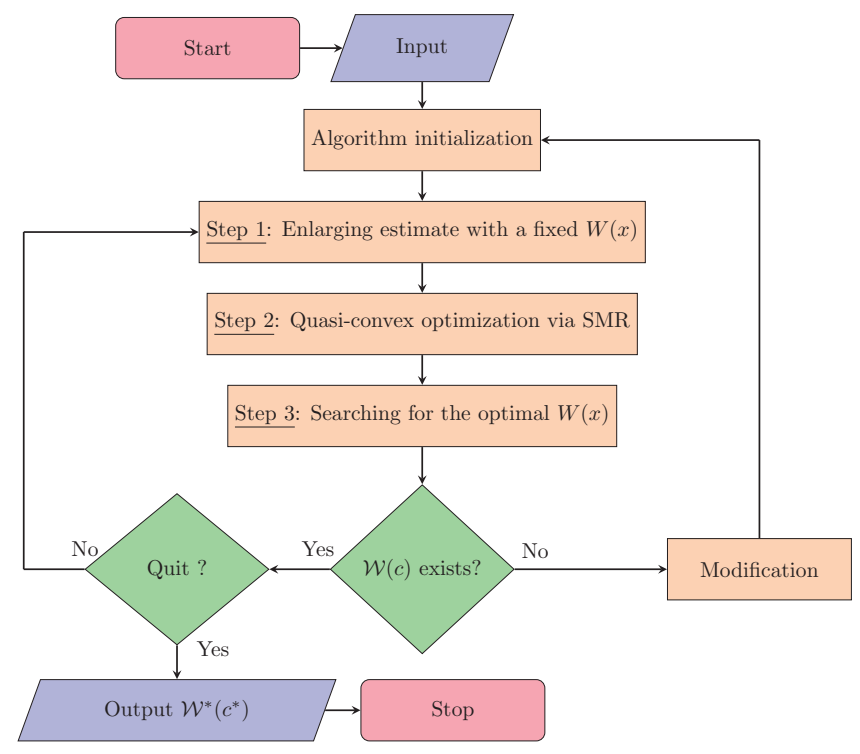

Fig. 4. Algorithm flowchart of the proposed approach.

\section{B. Step 1: Estimation with Fixed $W(x)$}

In this subsection, a method based on SOS programming is proposed to enlarge the set $\mathcal{W}(c)$ by selecting a fixed $W(x)$, i.e., we aim at finding

$$
\gamma=\sup c
$$

such that (6) holds.

To this end, we consider autonomous nonlinear systems with polynomial vector fields. This class of systems can be extended to non-polynomial vector fields by using polynomial approximation or recasting them into rational vector fields [3], which is outside the scope of this paper.

First, let us introduce a lemma which extends from the well-known Real Positivstellensatz (P-satz) [7], [30]. This lemma uses the cone of local SOS to check the positivity of a polynomial on a semialgebraic set.

Lemma 2 ([27]): For polynomials $a_{1}, \ldots, a_{m}, b_{1}, \ldots, b_{l}$ 
and $p$, define a set

$$
\begin{aligned}
\mathcal{B}= & \left\{x \in \mathbb{R}^{n}: a_{i}(x)=0, \forall i=1, \ldots, m,\right. \\
& \left.b_{i}(x) \geq 0, \forall j=1, \ldots, l\right\} .
\end{aligned}
$$

Let $\mathcal{B}$ be compact. Condition $\forall x \in \mathcal{B}: p(x)>0$ can be established if

$$
\left\{\begin{array}{l}
\exists r_{1}, \ldots, r_{m} \in \mathcal{P}, s_{1}, \ldots, s_{l} \in \mathcal{P}_{0}^{\mathrm{SOS}} \\
p-\sum_{i=1}^{m} r_{i} a_{i}-\sum_{i=1}^{l} s_{i} b_{i} \in \mathcal{P}_{0}^{\mathrm{SOS}}
\end{array}\right.
$$

Remark 4: Condition (9) becomes a necessary and sufficient condition if there exists no degree limit for the auxiliary functions $s_{i}$ and if one could find a polynomial $b$ in set $\mathcal{B}$ such that $b^{-1}[0, \infty)$ is compact in $\mathbb{R}^{n}$. Please find [30] for the instance of SOS cones.

Based on Lemma 2, a condition of local SOS programming can be proposed to obtain a lower bound of $\gamma$ in (7).

Theorem 2: Assume there exist a natural number $k$ and a positive definite function $W(x)$ satisfying

$$
\left\{\begin{array}{l}
\forall x \in \mathbb{R}_{0}^{n}: W(x)>0, W\left(0_{n}\right)=0, \\
\lim _{\|x\| \rightarrow \infty} W(x)=\infty \\
W^{(j)}(x)=0, \forall j=0,1, \ldots, 2 k
\end{array}\right.
$$

and there exist a group of polynomials $r_{j}(x) \in \mathcal{P}, j=$ $0,1, \ldots, 2 k$, and a polynomial $s(x) \in \mathcal{P}_{0}^{\text {SOS }}$ such that $\bar{c}$ is the optimum of the following polynomial optimization

$$
\begin{aligned}
& \bar{c}=\sup _{c, r, s} \\
& \text { s.t. }\left\{\begin{array}{l}
-\psi(x, c, r(x), s(x)) \in \mathcal{P}_{0}^{\mathrm{SOS}}, \\
\forall x \in \mathcal{W}(c) \backslash\left\{0_{n}\right\},
\end{array}\right.
\end{aligned}
$$

where

$$
\begin{aligned}
\psi(x, c, r(x), s(x))= & W^{(2 k+1)}(x)+s(x)(c-W(x)) \\
& +\sum_{j=0}^{2 k} r_{j}(x) W^{(j)}(x),
\end{aligned}
$$

in which $r(x)=\left[r_{0}(x), r_{1}(x), \ldots, r_{2 k}(x)\right]^{T}$. We recall that $W^{(j)}$ is the $j$-th derivative of $W$ with $W^{(0)} \equiv 0$. Then, $\bar{c} \leq \gamma$.

Proof: Suppose (11) holds, then $-\psi(x, c, s(x))$ and $s(x)$ are local SOS. From Lemma 2, it yields that

$$
\exists k: W^{(2 k+1)}(x)<0,
$$

for all $x$ in $\left\{x \in \mathbb{R}^{n}: W^{(j)}=0, j=0,1, \ldots 2 k\right\} \bigcap\{x \in$ $\left.\mathbb{R}^{n}: c-W(x) \geq 0\right\} \backslash\left\{0_{n}\right\}$, i.e., (6) holds. Therefore, from Theorem $1, \mathcal{W}(\bar{c})$ is an estimate of the DA with repect to some natural number $k$. Taking into account the definition of $\gamma$ in (7), it finally yields that $\bar{c}$ is a lower bound of $\gamma$, which completes this proof.

Remark 5: The above result converts the condition of (6) to local SOS conditions by using Lemma 2. It paves the way for proposing more tractable conditions via LMIs. The conservatism level depends on the degree of auxiliary functions and the existence of some additional auxiliary functions relaxed from P-satz, see [9], [12], [14].

\section{Step 2: Quasi-Convex Optimization via SMR}

Notice that the condition (11) of Theorem 2 is usually hard to establish since the product of a local SOS $s(x)$ and a scalar $c$ makes it a bilinear inequality, which is non-convex in nature. Bisection methods and iteration algorithms of SOS programming are introduced to cope with this issue, but they cannot handle the local SOS directly.

In this subsection, the SMR method is adopted for the local SOS, i.e., $p_{0}(x) \in \mathcal{P}_{0}^{\text {SOS }}$, and an approach is proposed by transforming the problem (11) into a generalized eigenvalue problem. First we will introduce the SMR for the set of local SOS. Consider a polynomial $p_{0}(x)$ of degree $\operatorname{deg}\left(p_{0}\right)$ without the constant and linear terms, or $p_{0}(x) \in \mathcal{P}_{0}^{\mathrm{SOS}}$. For this class of polynomial, its SMR is as follows:

$$
p_{0}(x)=(*)^{T}\left(\bar{P}_{0}+L(\delta)\right) \phi\left(n, d_{p_{0}}\right),
$$

where $(*)^{T} A B$ is short for $B^{T} A B$ given in Section II; $\bar{P}_{0}$ is denoted by the SMR matrix of $p_{0}(x) ; n$ is the number of variables; $d_{p_{0}}$ is the smallest integer not less than $\frac{\operatorname{deg}\left(p_{0}\right)}{2}$, i.e., $d_{p_{0}}=\left\lceil\frac{\operatorname{deg}\left(p_{0}\right)}{2}\right\rceil ; \phi\left(n, d_{p_{0}}\right) \in \mathbb{R}^{l\left(n, d_{p_{0}}\right)}$ is a power vector containing all monomials of degree less or equal to $d_{p_{0}}$ without degree 0 ; and $L(\delta)$ is a parameterization of the space

$$
\begin{aligned}
\mathscr{L}= & \left\{L(\delta) \in \mathbb{R}^{l\left(n, d_{p_{0}}\right) \times l\left(n, d_{p_{0}}\right)}: L(\delta)=L^{T}(\delta),\right. \\
& \left.(*)^{T} L(\delta) \phi\left(n, d_{p_{0}}\right)=0\right\},
\end{aligned}
$$

in which $\delta \in \mathbb{R}^{\vartheta\left(n, d_{p_{0}}\right)}$ is a vector of free parameters. The functions of $l\left(n, d_{p_{0}}\right)$ and $\vartheta\left(n, d_{p_{0}}\right)$ can be obtained similarly to [12]. For the ease of understanding, an illustration of an $\mathrm{SMR}$ is given.

Example 2: Given the polynomial $p_{1}(x)=5 x^{6}+4 x^{5}+$ $4 x^{4}+7 x^{2}$, we have $d_{p_{1}}=3, n=1$ and $\phi\left(n, d_{p_{1}}\right)=$ $\left(x^{3}, x^{2}, x\right)^{T}$. Then, $p_{1}(x)$ can be written as follows,

$$
P=\left(\begin{array}{ccc}
5 & 2 & 0 \\
2 & 4 & 0 \\
0 & 0 & 7
\end{array}\right), L(\delta)=\left(\begin{array}{ccc}
0 & 0 & -\delta \\
0 & 2 \delta & 0 \\
-\delta & 0 & 0
\end{array}\right) \text {. }
$$

Define $q(x)=\sum_{j=0}^{2 k} r_{j}(x) W^{(j)}(x)$ and let $\operatorname{deg}\left(W^{(2 k+1)}\right)-\operatorname{deg}(W) \leq \operatorname{deg}(s)$ and $\operatorname{deg}\left(W^{(2 k+1)}\right)-$ $\operatorname{deg}\left(W^{(j)}\right) \leq \operatorname{deg}\left(r_{j}\right)$, for all $j=0,1, \ldots, 2 k$. By exploiting the representation introduced in (14), we have the following expressions of SMR:

$$
\begin{aligned}
W(x) & =(*)^{T} \bar{W} \phi\left(n, d_{w}\right), \\
s(x) & =(*)^{T} \bar{S} \phi\left(n, d_{s}\right), \\
r_{j}(x) & =(*)^{T} \bar{R}_{j} \phi\left(n, d_{r_{j}}\right), \\
\psi(x) & =(*)^{T} \bar{\Psi}\left(\delta, c, \bar{R}_{j}, \bar{S}\right) \phi\left(n, d_{\psi}\right),
\end{aligned}
$$

for $j=0,1, \ldots, 2 k$, where $\delta \in \mathbb{R}^{\vartheta\left(n, d_{\psi}\right)}$ is a vector of free parameters, and $\bar{W} \in \mathbb{R}^{l\left(n, d_{w}\right) \times l\left(n, d_{w}\right)}, \bar{S} \in \mathbb{R}^{l\left(n, d_{s}\right) \times l\left(n, d_{s}\right)}$ and $\bar{\Psi}(\delta, c, \bar{Q}, \bar{S}) \in \mathbb{R}^{l\left(n, d_{\psi}\right) \times l\left(n, d_{\psi}\right)}$ are symmetric matrices. Let $\bar{D}(\delta), \bar{Q}\left(\bar{R}_{j}\right), \Lambda_{1}(S)$ and $\Lambda_{2}(S)$ be SMR matrices of $W^{(2 k+1)}(x), q(x), s(x)$ and $W(x) s(x)$, respectively, with respect to the power vector $\phi\left(n, d_{\psi}\right)$. From (12), it yields

$$
\Psi\left(\delta, c, \bar{R}_{j}, \bar{S}\right)=\bar{D}(\delta)+\bar{Q}\left(\bar{R}_{j}\right)+c \Lambda_{1}(\bar{S})-\Lambda_{2}(\bar{S}),
$$


where $\delta \in \mathbb{R}^{\vartheta\left(n, d_{\psi}\right)}$ is a vector of free parameters. The following result transforms the condition (11) into a generalized eigenvalue problem (GEVP) via SMR.

Theorem 3: For the given positive scalars $\varphi_{1}, \varphi_{2}$, a natural number $k$, and a selected polynomial $W(x)=$ $(*)^{T} \bar{W} \phi\left(n, d_{w}\right)$ fulfilling (10), the polynomial $\varsigma(x)=$ $\varphi_{1} s(x)+\varphi_{2} W(x) s(x)=(*)^{T} \Lambda(\bar{S}) \phi\left(n, d_{\psi}\right)$, the lower bound of $\gamma$ can be obtained by

$$
\tilde{\gamma}=-\frac{\tilde{e}}{\varphi_{1}+\varphi_{2} \tilde{e}}
$$

where $\tilde{e}$ is the solution of the GEVP

$$
\begin{aligned}
& \tilde{e}=\inf _{\delta,} e, \bar{R}_{j}, \bar{S} \\
& \text { s.t. }\left\{\begin{array}{l}
\varphi_{1}+\varphi_{2} e>0, \\
\bar{S}>0, \\
e \Lambda(\bar{S})>\bar{D}(\delta)+\bar{Q}\left(\bar{R}_{j}\right)-\Lambda_{2}(\bar{S}) .
\end{array}\right.
\end{aligned}
$$

Proof: In this proof, we first show that 1) (21) is a GEVP. Then, we demonstrate 2) (20) is the lower bound of $\tilde{\gamma}$.

1) Optimization (21) is a GEVP: From [27, Lemma 4], we have $\Lambda>0$ on the condition that $\bar{W}>0$ and $\bar{S}>0$, which makes (21) a GEVP.

2) $\tilde{\gamma}$ in (20) is the lower bound of $\tilde{\gamma}$ : Based on the last inequality of (21), we have

$$
\begin{aligned}
\widetilde{\Phi}\left(\delta, c, \bar{R}_{j}, \bar{S}\right)= & \bar{D}(\delta)+\bar{Q}\left(\bar{R}_{j}\right) \\
& -e \Lambda(\bar{S})-\Lambda_{2}(\bar{S}) \\
< & 0
\end{aligned}
$$

Considering (19) and

$$
\begin{aligned}
\tilde{\psi}(x, c, r(x), s(x))= & W^{(2 k+1)}(x)+q(x)-W(x) s(x) \\
& -e\left(\varphi_{1}+\varphi_{2} W(x)\right) s(x)
\end{aligned}
$$

one can rewrite $\tilde{\psi}(x, c, r(x), s(x))$ into:

$$
\tilde{\psi}(x, c, r(x), s(x))=\tilde{\psi}\left(x, r(x), \frac{-e}{\varphi_{1}+\varphi_{2} e},\left(\varphi_{1}+\varphi_{2} e\right) s(x)\right) \text {. }
$$

Notice that $-e /\left(\varphi_{1}+\varphi_{2} e\right)$ is a monotonically decreasing function which maps from the range $\left(-\left(\varphi_{1} / \varphi_{2}\right), 0\right]$ into the range $[0,+\infty)$. Thus, (20) gives the lower bound of $\tilde{\gamma}$.

For more details of the GEVP, please see the book [31].

\section{Step 3: Estimation with Optimal $W(x)$}

In this subsection, strategies for finding the optimal $W(x)$ are presented ${ }^{2}$. First, let us recall that $\rho$ in problem (4) is a user-selected measure which is usually chosen as

$$
\rho(\mathcal{W}(\gamma))=\operatorname{vol}(\mathcal{W}(\gamma))
$$

where $\operatorname{vol}(\mathcal{W}(\gamma))$ depicts the volume of $\mathcal{W}(\gamma)$, and $\gamma$ is introduced in (7). This allows us to pursue the optimal $W(x)$ via maximizing the volume of $\mathcal{W}(\gamma)$. However, $\operatorname{vol}(\mathcal{W}(\gamma))$ is highly non-convex, which makes (4) a non-convex optimization. To solve this problem, a typical method is to approximate $\operatorname{vol}(\mathcal{W}(\gamma))$ by introducing

$$
\omega=\max \frac{\gamma^{n}}{\operatorname{det}(\bar{W})}, \operatorname{vol}(\mathcal{W}(\gamma)) \propto \omega,
$$

${ }^{2}$ For each iteration, the optimal $W(x)$ is computed for all $k \in$ $\left\{0,1, \ldots, k_{d}\right\}$ where $k_{d}$ is a user-defined positive integer. where $\bar{W}$ is the SMR matrix of $W(x)$ in (16) and $\operatorname{vol}(\mathcal{W}(\gamma))$ is proportional to $\omega$. Then, a linear approximation of $\operatorname{vol}(\mathcal{W}(\gamma))$ can be provided as

$$
\operatorname{vol}(\mathcal{W}(\gamma)) \approx \frac{\gamma}{\operatorname{trace}(\bar{W})}
$$

The main idea is to minimize $\operatorname{det}(\bar{W})$ (the product of positive eigenvalues) by approximating the minimum of $\operatorname{trace}(\bar{W})$ (the sum of positive eigenvalues). Thus, a strategy is given for searching the optimal $W(x)$.

Proposition 1: Assume that there exist polynomials $\tilde{s} \in$ $\mathcal{P}^{\text {SOS }}, s \in \mathcal{P}_{0}^{\mathrm{SOS}}$ and $r_{j} \in \mathcal{P}, \forall j=0,1, \ldots, 2 k$, such that

$$
\begin{aligned}
& \zeta=\inf _{\bar{W}} \operatorname{trace}(\bar{W}) \\
& \text { s.t. }\left\{\begin{array}{l}
W(\bar{W}, x) \in \mathcal{P}_{0}^{\mathrm{SOS}}, W^{j}(\bar{W}, x)=0, \\
-\psi(x, \bar{W}, c, r(x), s(x)) \in \mathcal{P}_{0}^{\mathrm{SOS}} .
\end{array}\right.
\end{aligned}
$$

Then, $\mu_{1}=\frac{\gamma}{\zeta}$ is an under-estimate of $\rho$.

Note that the condition of (24) could be transformed to LMIs by using SMR introduced in the previous subsection.

Another promising method for handling the variable $W(x)$ is to enlarge $\mathcal{W}$ by using a set of chosen geometric shapes [14]. Specifically, we consider the optimization problem:

$$
\begin{aligned}
& \mu_{2}=\sup _{W, \epsilon} \epsilon \\
& \text { s.t. }\left\{\begin{array}{l}
\mathcal{G}(\epsilon) \subseteq \mathcal{W}(\gamma), \\
(10)-(11) \text { hold. }
\end{array}\right.
\end{aligned}
$$

where

$$
\mathcal{G}(\epsilon)=\left\{x \in \mathbb{R}^{n}: \mathscr{G}(x) \leq \epsilon\right\}
$$

and $\mathscr{G}(x)$ is a shape factor, e.g., a spherical form can be obtained from $\left\{x \in \mathbb{R}^{n}: \mathscr{G}(x) \leq \epsilon\right\}$ by simply choosing $\mathscr{G}(x)=\|x\|^{2}$. Analogous to Theorem 2, another strategy for computing the lower bound of $\rho(\mathcal{W}(c))$ can be given as follows:

Proposition 2: Assume that there exist polynomials $\tilde{s} \in$ $\mathcal{P}^{\mathrm{SOS}}, s \in \mathcal{P}_{0}^{\mathrm{SOS}}$ and $r_{j} \in \mathcal{P}, \forall j=0,1, \ldots, 2 k$, such that

$$
\begin{aligned}
& \mu_{2}=\sup _{W, \epsilon, c} \epsilon \\
& \text { s.t. }\left\{\begin{array}{l}
(\gamma-W)-\tilde{s}(\epsilon-\mathscr{G}) \in \mathcal{P}^{\mathrm{SOS}}, \\
W \in \mathcal{P}_{0}^{\mathrm{SOS}}, W^{j}(x)=0, \\
-\psi(x, c, r(x), s(x)) \in \mathcal{P}_{0}^{\mathrm{SOS}} .
\end{array}\right.
\end{aligned}
$$

Then, $\mu_{2}$ is an under-estimate of $\rho$.

To cope with (27), one could also construct a GEVP as in Theorem 3. We will show both strategies by numerical examples in the next section.

\section{EXAMPLES}

We execute the computation using MATLAB 2015a on a standard laptop with an 8GB DDR3 RAM and an Intel Core i7-4712MQ processor. MATLAB toolboxes SeDuMi, SDPT3, SMRSOFT and SOSTOOLS are used for solving semi-definite problems and SOS programming problems. 


\section{A. Example 3}

Consider the following model from [15]:

$$
\begin{aligned}
& \dot{x}_{1}=x_{2}, \\
& \dot{x}_{2}=-\left(1-x_{1}^{2}\right) x_{1}-x_{2},
\end{aligned}
$$

where the origin $0_{2}$ is a locally asymptotically stable equilibrium point with an unbounded DA.

First, we apply the algorithm shown in Fig. 3 and use Proposition 1 to compute the estimate of the DA. The proposed method is compared to the polynomial Lyapunov methods from [3], [27], by using the same enlarging strategy. From Fig. 5, it is not hard to see that the proposed method, which uses invariant sets constructed by Theorem 1, provides the best result compared to the other methods. Furthermore, a method comparison is also provided by using Proposition 2, as shown in Fig. 6. By selecting the shape factor $\mathcal{G}(x)=$ $x_{1}^{2}+x_{2}^{2}$, the best estimate can be obtained by the proposed method compared to the polynomial Lyapunov function methods. TABLE I shows the computational complexity of the proposed method.

\section{B. Example 4}

We extend the implementations to a 3-dimensional system:

$$
\begin{aligned}
& \dot{x}_{1}=-x_{1}+x_{2} x_{3}, \\
& \dot{x}_{2}=-x_{2}+x_{1} x_{2}, \\
& \dot{x}_{3}=-x_{3},
\end{aligned}
$$

in which the origin $0_{3}$ is locally asymptotically stable. By using the proposed algorithm with Strategy 1, we have the computational result shown in Fig. 7. It shows that the proposed method provides a better performance compared to the sublevel set method via polynomial Lyapunov functions [3], [27].

\section{CONCLUSION AND Discussion}

An approach is provided for estimating the DA of autonomous polynomial systems by using a new type of invariant sets. This method supplies a complementary perspective to the sublevel set method of Lyapunov functions, and provides an expanded scope for searching the Lyapunov function. Firstly, based on the Barbashin-Krasovskii-LaSalle invariant principle, a sufficient condition is provided that a sublevel set of a polynomial $W(x)$ is an inner-approximation of the DA by checking the higher-order derivatives of $W(x)$. Secondly, the largest estimation of the DA can be obtained by solving a local SOS programming problem. This problem is then transformed to a generalized eigenvalue problem by using the Square Matrix Representation. Finally, two strategies are proposed for searching the optimal $W(x)$, and an iterative algorithm is provided to obtain the largest estimate of the DA via invariant sets. The examples demonstrate the effectiveness of the proposed method compared to the polynomial Lyapunov function methods.

Along with Remark 3, the main conservatism of this approach stems from the fact that only a suboptimal solution could be obtained for the GEVP (21), and the fact that (23) approximates the volume of sublevel set of $W(x)$, which is

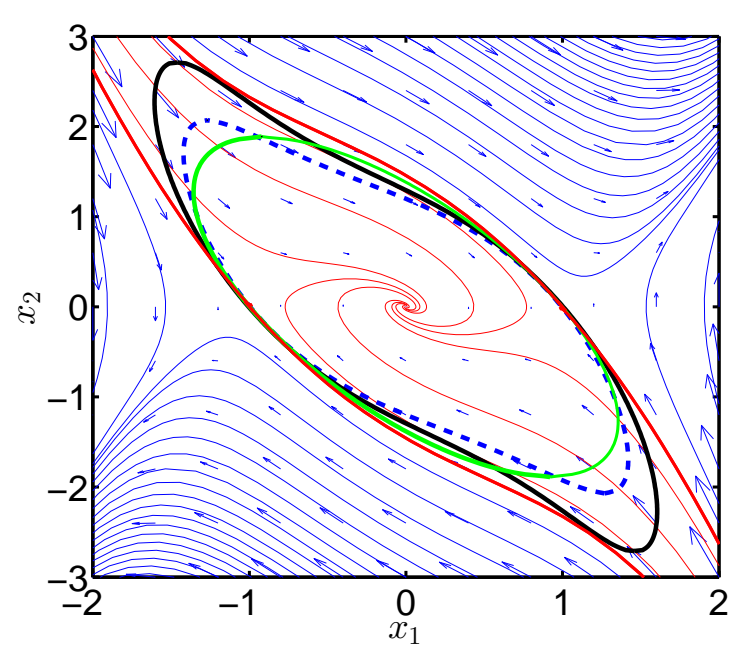

Fig. 5. Example 3: The computational result by using Proposition 1. The red solid line indicates the actual DA. The solid green line and the dotted blue line indicate the estimates by using the optimal Lyapunov functions with $\operatorname{deg}(V)=4$ and $\operatorname{deg}(V)=6$, perspectively. The solid black line depicts the estimate via the proposed method with $k=1$ and $\operatorname{deg}(W)=6$. Some trajectories are shown in red (converging) and blue (diverging).

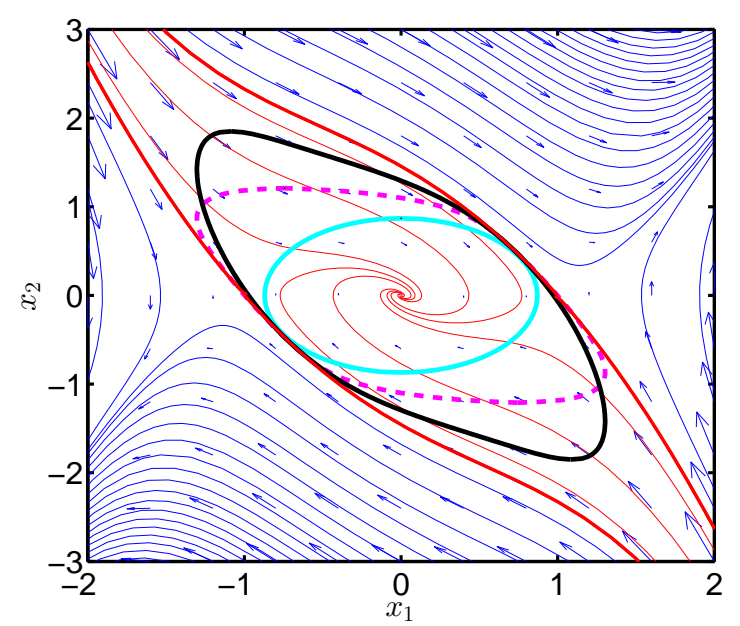

Fig. 6. Example 3: The computational result by using Proposition 2. The cyan solid line indicates the selected shape of the sublevel set of $\mathcal{G}$ from (26). The magenta dotted line indicates the estimates by using the optimal Lyapunov functions with $\operatorname{deg}(V)=6$. The solid black line depicts the estimate via proposed method with $k=2$ and $\operatorname{deg}(W)=6$.

TABLE I

THE COMPUTATIONAL TIME $t_{c}$ [sec] FOR THE DIFFERENT STRATEGIES, THE NUMBER OF ITERATION $n_{\mathrm{t}}$, AND THE DEGREE OF $W(x)$.

\begin{tabular}{llllllll}
\hline & \multicolumn{3}{c}{$\operatorname{deg}(W)=4$} & & \multicolumn{3}{c}{$\operatorname{deg}(W)=6$} \\
\cline { 2 - 3 } \cline { 6 - 8 } & $n_{\mathrm{t}=5}$ & $n_{\mathrm{t}}=10$ & $n_{\mathrm{t}}=20$ & & $n_{\mathrm{t}}=5$ & $n_{\mathrm{t}}=10$ & $n_{\mathrm{t}}=20$ \\
\hline Proposition 1 & 16.17 & 37.51 & 81.17 & & 30.32 & 69.91 & 151.47 \\
Proposition 2 & 19.83 & 37.92 & 87.36 & & 34.16 & 75.28 & 162.66 \\
\hline
\end{tabular}




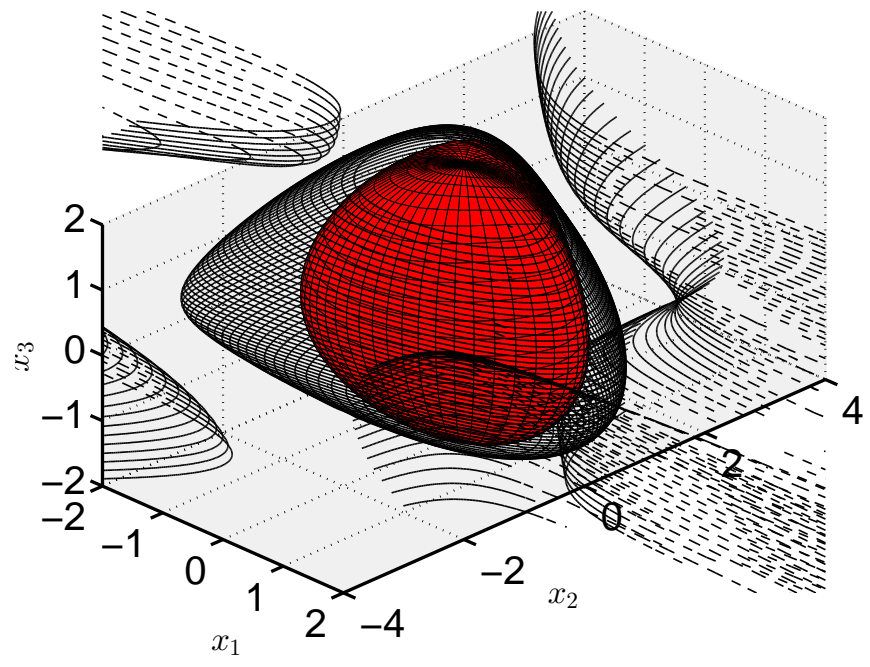

Fig. 7. Example 4: The computational result. The red region depicts the estimate of DA using polynomial Lyapunov functions with $\operatorname{deg}(V)=4$. The dotted black lines depict $\dot{V}(x)=0$. The solid black lines indicate the estimate via proposed method with $k=1$ and $\operatorname{deg}(W)=4$.

highly non-convex. To cope with these problems, a promising convex approach is proposed by using the moment theory and the occupation measure. Conditions of LMIs are obtained other than a bilinear matrix inequality (BMI) [23], [24]. Another source of conservatism arises from the fact that only inner-approximation of the exact DA can be obtained. To get less conservative results, using rational polynomial methods as well as the union of a group of sublevel sets are probable ways, to which our future efforts will be devoted [15], [16], [32]. Moreover, reachable set computations also provide a useful perspective to reduce the conservatism level [33].

\section{ACKOWLEDGements}

Financial support from the German Research Foundation (DFG AL 1185/2-1) is gratefully acknowledged.

\section{REFERENCES}

[1] R. Genesio, M. Tartaglia, and A. Vicino, "On the estimation of asymptotic stability regions: State of the art and new proposals," IEEE Transactions on Automatic Control, vol. 30, no. 8, pp. 747-755, 1985.

[2] A. I. Doban and M. Lazar, "Domain of attraction computation for tumor dynamics," in Proceedings of the Conference on Decision and Control, 2014, pp. 6987-6992.

[3] G. Chesi, Domain of attraction: Analysis and control via SOS programming. Springer Science \& Business Media, 2011, vol. 415.

[4] V. I. Zubov and L. F. Boron, Methods of A. M. Lyapunov and their Application. Noordhoff Groningen, 1964.

[5] G. P. Szegö, "On a new partial differential equation for the stability analysis of time invariant control systems," Journal of the SIAM, Series A: Control, vol. 1, no. 1, pp. 63-75, 1962.

[6] A. Vannelli and M. Vidyasagar, "Maximal Lyapunov functions and domains of attraction for autonomous nonlinear systems," Automatica, vol. 21, no. 1, pp. 69-80, 1985.

[7] J. Bochnak, M. Coste, and M.-F. Roy, Real algebraic geometry. Springer, 1998.

[8] D. Henrion and A. Garulli, Positive polynomials in control. Springer Science \& Business Media, 2005, vol. 312.

[9] P. A. Parrilo, "Structured semidefinite programs and semialgebraic geometry methods in robustness and optimization," Ph.D. dissertation, California Institute of Technology, 2000.
[10] S. Prajna, A. Papachristodoulou, P. Seiler, and P. A. Parrilo, "New developments in sum of squares optimization and SOSTOOLS," in Proceedings of the American Control Conference, 2004, pp. 56065611.

[11] A. Papachristodoulou and S. Prajna, "A tutorial on sum of squares techniques for systems analysis," in Proceedings of the American Control Conference, 2005, pp. 2686-2700.

[12] G. Chesi, "LMI techniques for optimization over polynomials in control: a survey," IEEE Transactions on Automatic Control, vol. 55, no. 11 , pp. 2500-2510, 2010.

[13] C. W. Scherer and C. W. J. Hol, "Matrix sum-of-squares relaxations for robust semi-definite programs," Mathematical programming, vol. 107, no. 1-2, pp. 189-211, 2006.

[14] Z. Jarvis-Wloszek, R. Feeley, W. Tan, K. Sun, and A. Packard, "Some controls applications of sum of squares programming," in Proceedings of the Conference on Decision and Control, 2003, pp. 4676-4681.

[15] W. Tan and A. Packard, "Stability region analysis using polynomial and composite polynomial Lyapunov functions and sum-of-squares programming," IEEE Transactions on Automatic Control, vol. 53, no. 2, pp. 565-570, 2008.

[16] G. Chesi, "Estimating the domain of attraction via union of continuous families of Lyapunov estimates," Systems \& Control Letters, vol. 56, no. 4, pp. 326-333, 2007.

[17] F. Amato, F. Calabrese, C. Cosentino, and A. Merola, "Stability analysis of nonlinear quadratic systems via polyhedral Lyapunov functions," in Proceedings of the American Control Conference, 2008, pp. 2291-2296.

[18] P. G. S. Hafstein, "Existence of piecewise affine Lyapunov functions in two dimensions," Journal of Mathematical Analysis and Applications, vol. 371, no. 1, pp. 233-248, 2010.

[19] G. Chesi, "Rational Lyapunov functions for estimating and controlling the robust domain of attraction," Automatica, vol. 49, no. 4, pp. 10511057, 2013.

[20] B. Tibken, "Estimation of the domain of attraction for polynomial systems via LMIs," in Proceedings of the Conference on Decision and Control, vol. 4, 2000, pp. 3860-3864.

[21] G. Valmorbida, S. Tarbouriech, and G. Garcia, "Region of attraction estimates for polynomial systems." in Proceedings of the Conference on Decision and Control, 2009, pp. 5947-5952.

[22] U. Topcu, A. Packard, and P. Seiler, "Local stability analysis using simulations and sum-of-squares programming," Automatica, vol. 44, no. 10 , pp. $2669-2675,2008$.

[23] D. Henrion and M. Korda, "Convex computation of the region of attraction of polynomial control systems," IEEE Transactions on Automatic Control, vol. 59, no. 2, pp. 297-312, 2014.

[24] M. Korda, D. Henrion, and C. Jones, "Inner approximations of the region of attraction for polynomial dynamical systems," in Proceedings of the IFAC Symposium on Nonlinear Control Systems, 2008, pp. 2291-2296.

[25] T.-C. Wang, S. Lall, and T.-Y. Chiou, "Polynomial method for PLL controller optimization," Sensors, vol. 11, no. 7, pp. 6575-6592, 2011.

[26] G. Valmorbida and J. Anderson, "Region of attraction analysis via invariant sets," in Proceedings of the American Control Conference, 2014, pp. 3591-3596.

[27] D. Han and M. Althoff, "Control synthesis for non-polynomial systems: a domain of attraction perspective," in Proceedings of the Conference on Decision and Control, 2015, pp. 1160-1167.

[28] H. K. Khalil and J. W. Grizzle, Nonlinear systems. Prentice hall Upper Saddle River, 2002.

[29] G. H. Ryder and M. D. Bennett, "Vibrations of single-degree-offreedom systems," in Mechanics of Machines. Springer, 1990, pp. 262-281.

[30] M. Putinar, "Positive polynomials on compact semi-algebraic sets," Indiana University Mathematics Journal, vol. 42, no. 3, pp. 969-984, 1993.

[31] S. Boyd, L. El Ghaoui, E. Feron, and V. Balakrishnan, Linear matrix inequalities in system and control theory. Society for industrial and applied mathematics, 1994.

[32] D. Han, A. El-Guindy, and M. Althoff, "Power systems transient stability analysis via optimal rational Lyapunov functions," in Proceedings of the IEEE Power and Energy Society General Meeting, 2016, to appear.

[33] M. Althoff and B. H. Krogh, "Reachability analysis of nonlinear differential-algebraic systems," IEEE Transactions on Automatic Control, vol. 59, no. 2, pp. 371-383, 2014. 